\title{
Properties and applications of photoresponsive polymers
}

\author{
Masahiro Irie \\ Institute of Advanced Material Study, Kyushu University \\ Kasuga-Koen 6-1, Kasuga, Fukuoka 816, Japan
}

\begin{abstract}
Several proposals to construct artificial photoresponsive polymer systems have been described. Polyamides with azobenzene chromophores in the backbone reversibly changed the conformation by ultraviolet light irradiation (410> $\lambda>350 \mathrm{~nm}$ ), which causes the trans to $\mathrm{cis}$ isomerization of the chromophores. Aqueous solutions of poly(N-isopropylacrylamide) with pendant azobenzene chromophores showed a photostimulated phase separation on exposure to ultraviolet light. A large effect, a large turbidity change in this case, was induced by a small number of photons. Polyacrylamide gels with triphenylmethane leucocyanide groups reversibly dilated as much as 18 time by ultraviolet irradiation. Photodissociation of the chromophores generated the osmotic pressure difference between the gel and the outer solution, and the osmotic effect caused the gels to expand. When a rod-shaped gel was irradiated from one side, the gel showed a bending motion. Electric field accelerated the bending rate. Poly(N-isopropylacrylamide) gels with triphenylmethane leucocyanide groups underwent a discontinuous swelling-shrinking switching upon irradiation and removing ultraviolet light.
\end{abstract}

\section{INTRODUCTION}

Biological systems have developed various kinds of photoactive organs to adapt themselves to sunlight. Plants, for example, have evolved photosynthesis systems to utilize the light as an energy source. At the same time, they have developed photoresponsive systems which use the light to seek out optimum conditions for their life processes. In the latter systems, the light is used as information, such as phototropism, phototaxis or vision. As in biological systems, light can be utilized in polymer chemistry not only as an energy source for polymer synthesis but also as an information source or a trigger for the reversible control of the physical and chemical properties of polymers.

Although we are not yet able to reconstruct the biological photoresponsive systems, it is worthwhile mimicking the processes and applying them to the molecular design of synthetic photoresponsive polymers, so that the physical and chemical properties can be changed reversibly by photoirradiation. The feature common to the biological photoresponsive systems is that they contain photochromic molecules embedded in biopolymer matrices and use the photoisomerization for controlling the conformation and assembly of the biopolymers. The photostimulated structural change of the biopolymers perturbs the subsequent biochemical reactions.

In a similar manner to biological systems, photoresponsive polymers consists of a photoreceptor part, which contains photochromic molecules, and a functional part (see Fig. 1).

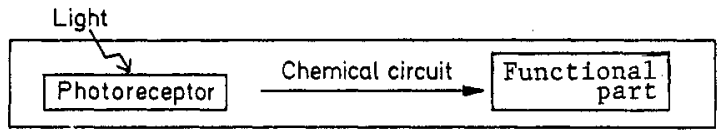

Fig. 1. Photoresponsive polymer

At first, an optical signal is captured by the photochromic chromophores and converts to a chemical signal owing to the photoisomerization. Then, the chemical signal is transferred to the functional part via a chemical circuit, and controls the polymer properties. In some cases, amplification mechanisms are involved. 
The number of photochromic chromophores used in the biological systems is limited, while in the synthetic photoresponsive polymers, the number of chromophores that can be used is limitless. Depending on the purpose, a suitable chromophore can be chosen from a great number of synthetic photochromic compounds. In addition, many aspects of polymer properties can be employed for the functional part. The purpose of the present research is to study the fundamental principles useful for constructing artificial photoresponsive polymers(ref.1).

\section{CONFORMATION CHANGE OF AZOBENZENE-CONTAINING POLYAMIDE}

Many photosensitive molecules are known to be transformed under photoirradiation into other isomers, which can return to the initial state either thermally or photochemically.

$$
\text { A } \stackrel{h \nu}{\rightleftharpoons} B
$$

These reactions are referred as photoisomerization. The chromophores capable of the reactions are called photochromic molecules. The reactions are always accompanied by certain changes in the physical and chemical properties of the chromophores, such as dipole moment and geometrical structure. These changes may drive the property changes of polymers in which the chromophores are incorporated.

A conformation is one of the characteristic properties of polymers in solution, and it controls various polymer properties. At first, the guiding principle for molecular design of polymers which change the conformation reversibly by photoirradiation will be given as the general model of photoresponsive polymers.

Figure 2 illustrates several proposals to use photochromic chromophores as a tool for conformation changes. Here, the most simple mechanism(1) will be described(ref. $2,3,4)$. When trans-cis photoisomerizable chromophores are incorporated into the polymer backbone, the photoinduced configuration change of the chromophores is expected to induce a conformation change of the polymer chain. Azobenzene( 1$)$ is the most widely used as the trans-cis photoisomerizable photoreceptor molecule. It undergoes isomerization from the trans to the cis form under ultraviolet irradiation, while the cis form can return to the trans form either thermally or photochemically.<smiles>c1ccc(/N=N/c2ccccc2)cc1</smiles>

(1)

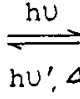

hu', 4

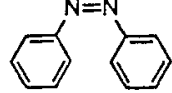

(2)

During the isomerization, the distance between 4 and $4^{\prime}$ carbons decreases from 9.0 to $5.5 \mathrm{~A}$. A typical example of the polymers having azobenzene chromophores is following polyamide( 3 ).<smiles>O=C(O)c1cc(C(=O)O)c(C(=O)Nc2ccc(N=Nc3ccc(NC4CC4)cc3)cc2)cc1C(=O)O</smiles>

The intrinsic viscosity of polyamide( $\underline{3})$ in polar $N, N$-dimethylacetamide was found to decrease from 1.22 to $0.50 \mathrm{dl} / \mathrm{g}$ upon ultraviolet irradiation( $410>\lambda>350 \mathrm{~nm}$ ) and to return to the initial value in $30 \mathrm{~h}$ in the dark at $20^{\circ} \mathrm{C}$. The slow recovery of the viscosity in the dark was accelerated by visible light irradiation ( $\lambda$ > $470 \mathrm{~nm}$ ).

1
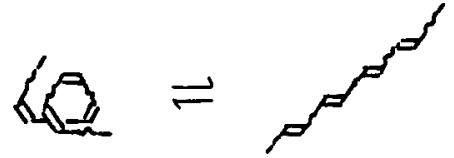

2.<smiles>CCCCCCCCCCCCC</smiles>
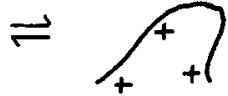

3

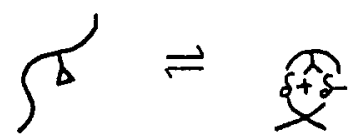

Fig. 2. Schematic illustration of photostimulated conformation changes of polymer chains.

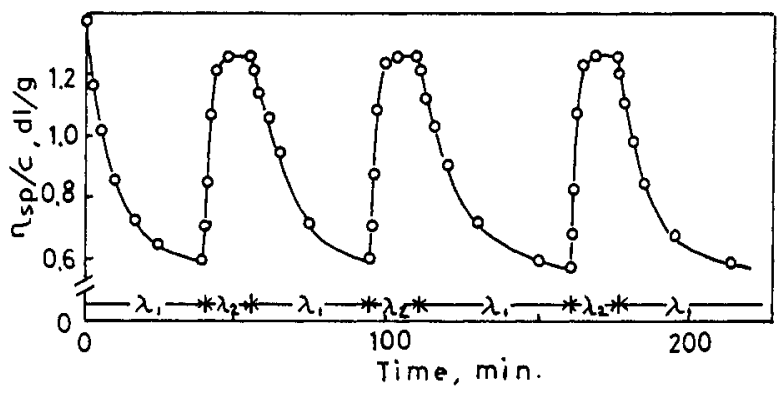

Fig. 3. Photostimulated viscosity change of $\mathrm{N}, \mathrm{N}-$ dimethylacetamide solution of polyamide(3) on alternate irradiation with ultraviolet light $(410>\lambda>350 \mathrm{~nm})$ and visible $\operatorname{light}(\boldsymbol{\lambda}>470 \mathrm{~nm})$ at $20^{\circ} \mathrm{C}$. Concentration of the polymer was 0.9 $g / a 1$. 
On alternate irradiation of ultraviolet and visible light, the viscosity reversibly changed as much as $60 \%$, as shown in Fig. 3. Before photoirradiation, the polyamide has a rod like conformation. The isomerization from the trans to the cis form kinks the polymer chain, resulting in a compact conformation and a decrease in the viscosity. The compact conformation returns to the initial extended conformation either thermally or visible light irradiation, thereby causing an increase in the viscosity.

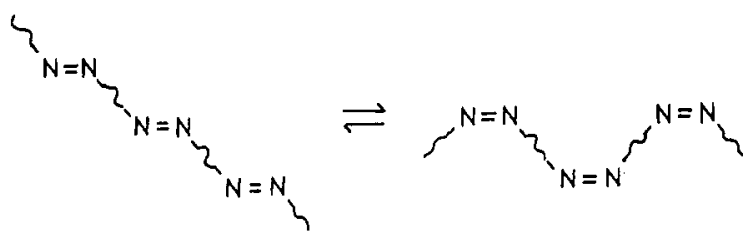

According to the above illustration, rigidity of the polymer chain is expected to alter the amount of photodecrease of the solution viscosity. When the azobenzene residues are connected by rigid phenylene groups, the resulting viscosity change should be large, while it should become small when the connecting groups are flexible, as long methylene chains. The effect of backbone structure on the photodecrease of solution viscosity is summarized in Table 1. As expected, the amount of photodecrease of the viscosity decreased with increasing number of methylene groups in the polymer backbone. The stiffest polymer, that having phenylene residues, gives a large photodecrease, while the viscosity of polymers having flexible long methylene chains is hardly reduced by photoirradiation. The absence of photodecrease in the polymer with 12 methylene groups suggests that flexible methylene chains act as a strain absorber. The conformation change induced by the isomerization of the azobenzene residues is relaxed in the connecting flexible methylene chains, resulting in no change of the shape of the polymer.

Table 1. Effect of backbone structure of the polymers on the photodecrease of the solution viscosity.

\begin{tabular}{lc}
\hline $\mathrm{R}^{\mathrm{a}}$ & $\begin{array}{r}\text { specific viscosity(uv)/ } \\
\text { specific viscosity(dark) }\end{array}$ \\
$-\mathrm{p}^{-\mathrm{C}_{6} \mathrm{H}_{4}{ }^{+}}$ & 0.37 \\
$-\mathrm{CH}_{2}{ }_{4}$ & 0.59 \\
$+\mathrm{CH}_{2}{ }_{8}$ & 0.80 \\
$-\mathrm{CH}_{2}{ }_{12}$ & 0.96 \\
\hline
\end{tabular}

a) $\mathrm{R}$ group in $+\mathrm{NH}-\mathrm{N}-\mathrm{N}-\mathrm{NHCO}-\mathrm{R}-\mathrm{CO} \mathrm{T}_{\mathrm{n}}$

Similar experiments have been carried out for polyamide(4) and ( $\underline{5}$ ) by Blair et al.(ref. 5). They could not, however, observe any decrease in the intrinsic viscosity under ultraviolet irradiation in contrast with our result.<smiles>CC1CN(OCc2cccc(N=Nc3ccc(OC(C)(F)F)cc3)c2)C(C)N(OCc2cccc(OC(C)(F)F)c2)C1C</smiles>

The absence of the photodecrease in the viscosity is possibly due to inclusion of flexible piperazine segments in the polymer chain. Polyurea with backbone azobenzene groups(ref. 6,7 ), and polyquinoline with backbone stilbene groups(ref. 8) also showed the photostimulated decrease in the intrinsic viscosity.

Photostimulated changes of electrostatic forces, mechanism(2), or dipole-dipole interactions, mechanism(3), also control the polymer conformations. The mechanism(2) will be described in the section of dilation of polymer gels.

\section{PHASE SEPARATION OF POLYMER SOLUTIONS}

The photoresponsive polymers described above change the conformation in proportion to the number of photons that they absorb. Physical and chemical properties associated with the conformation changes also vary with the number of absorbed photons. To make a sensitive photoresponsive polymer,i.e. one which responds more efficiently to a fewer photons, we 
have to introduce an amplification mechanism into the system. A convenient way to achieve this end is to utilize the phase transition of polymers.

At a temperature close to the phase transition temperature, the system is in an unstable state, and hence a small perturbation may bring about a large effect on it. When such a system is perturbed by photochromic reactions, the absorption of a few photons will induce a large property change.

Figure 4 shows a schematic illustration of the photostimulated phase transition from the state $\mathrm{X}$ to the state $\mathrm{Y}$. When the photochromic chromophores in the polymer chain are $\mathrm{A}$ isomers, the polymer changes the state at a temperature of $\mathrm{Ta}$. We assume that the phase transition temperature will rise to Tb when A isomers convert to $B$ isomers. Then, if the isomerization from the $A$ to the $B$ isomer can be induced by photoirradiation at temperature $T(T a<T<T b)$, the state will change isothermally at $T$ from $Y$ to $X$ by photoirradiation. In this system, a few photons can induce a marked change in such properties as phase separation in polymer solutions, gel-sol transition, and miscibility of polymer blends. Here, phase separation in polymer solutions will be described.

\section{Reversible phase separation of aqueous poly( $\mathbf{N}$-isopropylacrylamide) solution (ref. 7)}

Poly (N-isopropylacrylamide) in water has a lower critical temperature Tc at $31^{\circ} \mathrm{C}$. The solution is homogeneous and transparent below $31^{\circ} \mathrm{C}$, while it becomes turbid, and shows phase separation above $31^{\circ} \mathrm{C}$. Tc depends on the subtle balance between the hydrogen bond formation ability of the polymer with water and the intermolecular hydrophobic forces. The hydrophobic interaction is expected to be controlled by photoirradiation, when photochromic chromophores, which show a polarity change upon irradiation, are introduced into the polymer. Thus, the temperature at which this system undergoes phase separation are expected to be altered by photoirradiation. Such an attempt was made by incorporating azobenzene chromophores into the pendant groups.

Figure 5 shows the transmittance change at $750 \mathrm{~nm}$ which occurred when a 18 aqueous solution of poly(N-isopropylacrylamide) with 2.7 molez pendant azobenzene groups was heated. In the dark before photoirradiation, the solution begins to be turbid at $18.5^{\circ} \mathrm{C}$ and the transmittance decreases to one-half the initial value at $19.4^{\circ} \mathrm{C}$. Upon ultraviolet irradiation $(410>\lambda>350 \mathrm{~nm})$, the phase separation temperature rises to $26.0^{\circ} \mathrm{C}$. The photostimulated change of $\mathrm{TC}$ means that between 19.4 and $26.0^{\circ} \mathrm{C}$ ultraviolet irradiation solubilizes the polymer and the solution becomes transparent, while visible irradiation decreases the solubility of the polymer and leads to phase separation.

The maximum difference in phase separation temperature was observed at a very small azobenzene content of 2.7 mols. Below and above this content, the phase separation was not affected by photoirradiation. This fact indicates that the phase transition temperature depends on a subtle balance between the polymer's ability of hydrogen bond formation with water and the intermolecular hydrophobic force, as described above. The isomerization of a small number of azobenzene chromophores ( $2-3$ mols) affects the balance, resulting in an efficient phase separation. A large effect, a large turbidity change in this case, was induced by a small number of photons in the temperature range from 19.4 to $26.0^{\circ} \mathrm{C}$. Below 19.4 and above $26.0^{\circ} \mathrm{C}$, the photostimulated phase separation was not observed. These findings are consistent with the schematic illustration in Fig. 4.

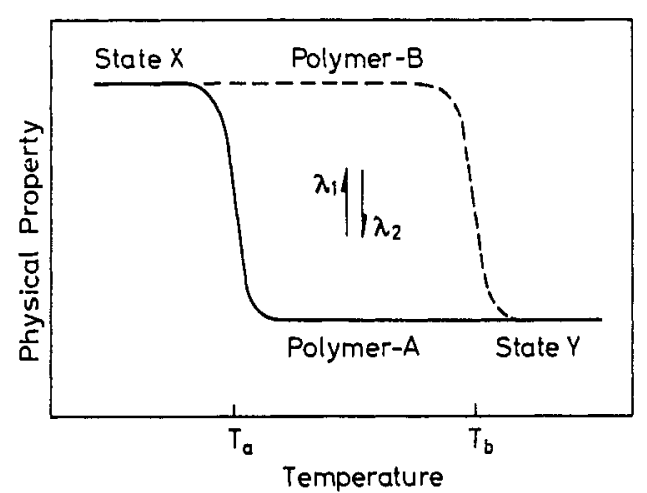

Fig. 4. Schematic illustration of photostimulated phase transition from the state $X$ to the state $Y$.

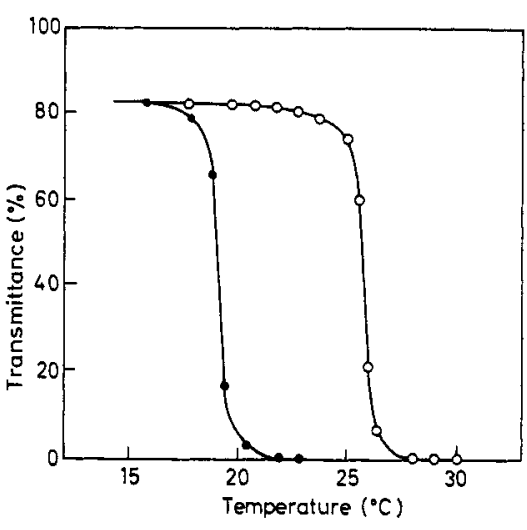

Fig. 5. Transmittance changes at $750 \mathrm{~nm}$ of $1 \&$ aqueous solution of poly( $\mathrm{N}-\mathrm{iso}-$ propylacrylamide) with pendant azobenzene groups $(2.7$ mols) when heated at a rate of $2 \mathrm{c} / \mathrm{min}$. ( $\bullet$ ) before photoirradiation; ( 0 ) under photostationary state with UV light $(410>\lambda>350 \mathrm{~nm})$. 


\section{Reversible solubility change of azobenzene-containing polystyrene in cyclohexane (ref. 10, 11)}

Similar phase separation was observed in theta solvents containing polymers with pendant photochromic chromophores. In a theta solvent, the interaction between the polymer and the solvent is in balance with intra- and inter-polymer interactions. The isomerization of the pendant chromophores alters this balance. A system examined was a cyclohexane solution of polystyrene with pendant azobenzene groups.

Cyclohexane becomes a theta solvent for polystyrene at $35^{\circ} \mathrm{C}$. Moderate molecular weight polystyrene $\left(\mathrm{MW} \sim 5 \times 10^{4}\right.$ ) with pendant azobenzene groups is soluble in this solvent at $30^{\circ} \mathrm{C}$. The solution became turbid upon ultraviolet irradiation(410> $\lambda>350 \mathrm{~nm}$ ). Prolonged irradiation caused the polymer to precipitate. The solution again became transparent when irradiated with visible light $(\lambda>470 \mathrm{~nm})$. The photoresponsive behavior is shown in Fig. 6 . The phase separation is ascribable to the isomerization of the pendant azobenzene groups. Introduction of non-polar trans form azobenzene chromophores into the pendant groups little affects the polymer-solvent interaction, while the photogenerated cis form tends to decrease the polymer-solvent interaction. Thus, upon ultraviolet irradiation, the polymer-solvent interaction decreases considerably until the polymer precipitates.

The precipitation behavior of the polymer is interpreted by a photostimulated change of the critical miscibility temperature TC. For polystyrene dissolved in cyclohexane, the polymer precipitates at temperature below TC. According Fox and Flory (ref. 12), Tc depends on the molecular weight $M$ as

$$
\mathrm{T}_{C}=\mathrm{Te}\left(1-\mathrm{b} / \mathrm{M}^{0.5}\right)
$$

where $T_{\ominus}$ is the value of $T C$ for $M=\infty$ and $b$ an empirical constant. We assume that $T_{\Theta}$ and $b$ change when the trans azobenzene groups convert to the cis form. Figure 7 shows a schematic illustration of the molecular weight dependence of TC.

First, we consider a mono-dispersed polystyrene of molecular weight M containing pendant azobenzene groups. When azobenzene groups are in the trans form, the polymex solution phase separates at $t_{t}$, which corresponds to $\mathrm{Ta}$ of Figure 2 . The isomerization of the chromophores from the trans to the cis form causes the phase separation temperature to rise to $t c$, which corresponds to Tb of Figure 2. This means that phase separation of the solution is induced between $t_{t}$ and $t_{c}$ by ultraviolet irradiation, which causes the trans-cis isomerization.

Next, we consider the phase separation of a poly-dispersed polystyrene with pendant azobenzene groups at constant temperature, $T_{M}$. With all azo groups in the trans configuration, any fractions of $\mathrm{M}<\mathrm{M} T$ are soluble in cyclohexane at $\mathrm{T}_{\mathrm{M}}$, but after photoirradiation, the fractions of $M c<M<M_{T}$ become insoluble and precipitate from the solution. Actually, irradiation here has the same effect as lowering the solution temperature from $\mathrm{T}_{\mathrm{M}}$ to $\mathrm{Tm}$. This process was successfully applied to the fractionation of poly-dipersed polystyrene(ref. 10,13 ).

The photoresponsive behavior described above is quite efficient. Only 5 mols azobenzene pendant groups were enough to cause the phase separation. In the system containing spirobenzopyran groups(ref. 13), the efficiency was much higher. The isomerization of 2 molo spirobenzopyran chromophores in the pendant groups raised TC considerably and led to phase separation.

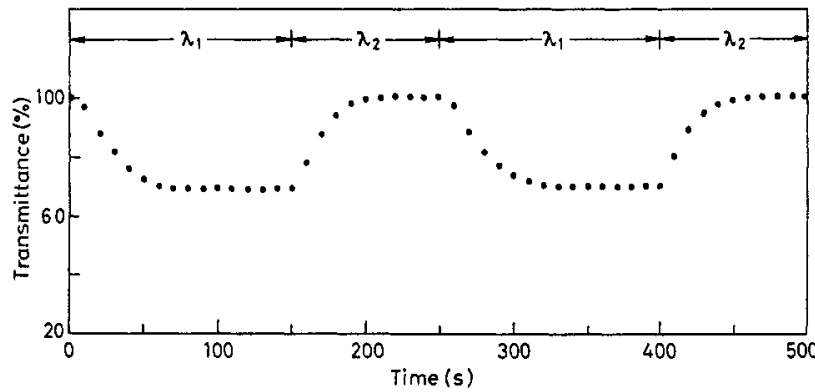

Fig. 6. Changes in transmittance at $650 \mathrm{~nm}$ of cyclohexane solution containing polystyrene with pendant azobenzene groups ( $6.1 \mathrm{~mol} \%$ ) on alternate irradiation with ultraviolet light $(410>\lambda>350 \mathrm{~nm})$ and visible $\operatorname{light}(\lambda>470 \mathrm{~nm})$ at $30^{\circ} \mathrm{C}$.

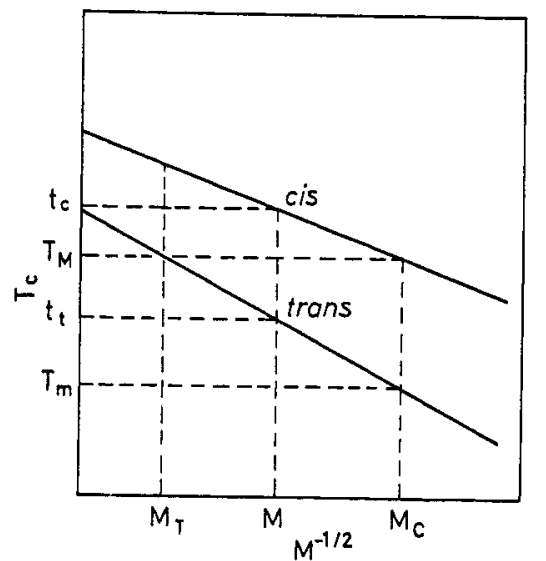

Fig. 7. Molecular weight(M) dependence of critical miscible temperature Tc. 


\section{DILATION OF POLYMER GELS}

The mechanism (2) shown in Fig. 2 uses the electrostatic repulsion between photogenerated charges as the driving force for conformation changes. The utility of this mechanism was examined by adopting triphenylmethane leucoderivatives( $\underline{6}$ ) as photoreceptor molecules (ref. 14). The chromophore dissociates into an ion-pair under ultraviolet irradiation, generating an intensely green colored triphenylmethyl cation. The cation thermally recombines the counter anion as follows.

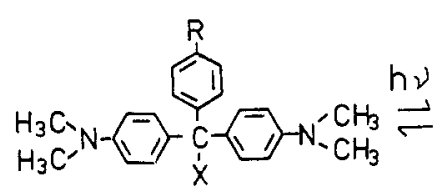

()

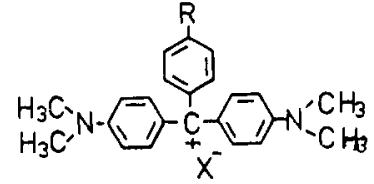

(7)

The triphenylmethane leucohydroxide residues were incorporated into the pendant groups by copolymerizing the vinyl derivative ( $\underline{6}, \mathrm{R}=\mathrm{CH}=\mathrm{CH}_{2}, \mathrm{X}=\mathrm{OH}$ ) with $\mathrm{N}, \mathrm{N}$-dimethylacrylamide. Upon ultraviolet irradiation $(\lambda>270 \mathrm{~nm})$, the solution became deep green, and at the same time its reduced viscosity increased from 0.55 to $1.6 \mathrm{dl} / \mathrm{g}$. After the light was shut of f; the viscosity as well as the absorption intensity returned to the initial value with a half-life of $3.1 \mathrm{~min}$. The result suggests that the electrostatic repulsive force caused the conformation to expand.

The concentration dependence of reduced viscosity confirmed the above expansion mechanism. In the dark before irradiation, the dependence was linear; the reduced viscosity decreased with decreasing the concentration of the polymer. During ultraviolet irradiation, this dependence showed anomalous behavior. The viscosity steeply increased at low polymer concentration. The viscosity during photoirradiation was 4 times larger than the viscosity in the dark at $0.04 \mathrm{~g} / \mathrm{dl}$. At low polymer concentration, screening of the electrostatic potential by the counter ions becomes weak, and consequently the increase of the repulsive forces of the positive charges along the polymer chain expands the dimension of chain. The photo-effect due to the electrostatic forces is much larger than the effect observed for polyamides having azobenzene residues in the backbone. Electrostatic repulsive force is effective to induce the conformation change of polymers.

\section{Photostimulated continuous volume change of polymer gels (ref. 15, 16)}

It is of particular interest to amplify the photostimulated conformation change of polymer chains in solution to a macroscopic change in the size of gels or solids. Although many systems showing photostimulated deformations have been reported, the deformations were limited to less than $10 \%$. Such a small deformation makes it difficult to judge whether the effect is due to photochemistry or photoheating. If the deformation is larger than $20 \%$, we may safely say that it is due to the photochemical effect. One photo-deformable material of interest is poly-4-(N,N-dimethylamino)-N-r-D-glutamanilide(ref. 17), which displayed a dilation amounting to 35 in $\mathrm{N}, \mathrm{N}$-dimethylformamide when exposed to light in the presence of $\mathrm{CBr}$. This large deformation was due to the ionization of $\mathrm{N}, \mathrm{N}$-dimethylanilide groups. Although the system was irreversible and no attempt was made to make it reversible, such a pronounced effect is informative for the design of reversible photo-deformable polymers.

We may infer from the studies on the conformation change in solution that the the electrostatic repulsion between photogenerated charges is more effective for conformation changes than the trans-cis isomerization of unsaturated linkages. On due consideration, we decided to take advantage of electrostatic forces to obtain gels exhibiting large reversible deformations. Acrylamide gels containing a small amount of triphenylmethane leucohydroxide or leucocyanide groups were prepared by free radical copolymerization of di(N,N-dimethylaniline)-4-vinylphenylmethane leuco hydroxide ( $\underline{\sigma}, \mathrm{x}=\mathrm{OH}, \mathrm{R}=\mathrm{CH}=\mathrm{CH}_{2}$ ) or leucocyanide ( $\underline{6}$, $\mathrm{X}=\mathrm{CN}, \mathrm{R}=\mathrm{CH}=\mathrm{CH}_{2}$ ) in dimethylsulfoxide in the presence of $\mathrm{N}, \mathrm{N}$-methylene-bisacrylamide.

The gels were swollen to equilibrium by allowing them to stand in water overnight. Then the changes in their weight and dimensions induced by ultraviolet light were measured. A diskshaped gel (10 mm in diameter and $2 \mathrm{~mm}$ in thickness) having 3.7 mols triphenylmethane leucohydroxide residues showed photostimulated reversible dilation in water. Figure 8 shows that upon ultraviolet irradiation $(\lambda>270 \mathrm{~nm})$, the gel swells and the weight increases as much as 3 times the original one in $1 \mathrm{~h}$. The dilated gel deswells in the dark to the initial weight in $20 \mathrm{~h}$. The cycles of dilation and contraction of the gel were repeated several times. The gel having leucohydroxide residues swelled even in the dark when the aqueous solution became acidic, owing to the chemical ionization of the residues. In order to make the gel insensitive to $\mathrm{pH}$ changes, the hydroxide residues were replaced by cyanide groups. The weight of the leucocyanide gel remained constant in the range of pH $4-9$. Upon ultraviolet light irradiation, the leuconitrile gel weight increases as much as 18 times. In the dark, the gel contracts again slowly to the initial weight. 
Triphenylmethyl cation is well known to have a very strong absorption at $622 \mathrm{~nm}$. Upon ultraviolet irradiation, the color of the gel changed quickly from pale green to deep green in less than 3 min and then remained almost constant. In the dark, the color returns to the initial pale green in several hours. The size of the gel, on the other hand, increased slowly and reached the saturated value in about $2 \mathrm{~h}$. The slow response of the size change in comparison with the color change indicates that the gel dilation is due not to the thermal effect but to the photochemical ionization. The formation of charges, fixed cations and free anions, generates an osmotic pressure difference between the gel and the outer solution, and this osmotic effect is considered to be responsible for gel expansion. It is worthwhile to note that the gel expansion is suppressed by the addition of salts, such as $\mathrm{NaCl}$ and $\mathrm{KBr}$. No photostimulated dilation was observed in the presence of $10^{-2} \mathrm{M} \mathrm{NaCl}$ or $\mathrm{KBr}$ for the gels having leucohydroxide or leucocyanide groups.

\section{Electric field effect (ref. 18, 19)}

The acrylamide gel described above is the first example showing a reversible deformation more than $100 \%$. The effect is purely photochemical and reversible. However, this gel has a serious disadvantage in that the response time is slow. To improve this point the effect of electric field on the gel deformation was examined. A rod-shaped acrylamide gel containing triphenylmethane leucocyanide groups(25 $\mathrm{mm}$ in length and $2 \mathrm{~mm}$ in section diameter) was prepared. The gel rod was inserted between two parallel platinum electrodes in a small water pool (Teflon, $36 \times 19 \times 15 \mathrm{~mm}$ ) and fixed at its one end on the pool wall.

Figure 9 shows photostimulated bending of an acrylamide gel having 3.1 molo triphenylmethane leucocyanide groups in water $(\mathrm{pH}$ 6.5) in an electric field. In the dark, the gel shape did not change in a $10 \mathrm{~V} / \mathrm{cm}$ electric field(Fig. 9a). Upon ultraviolet irradiation( $\lambda>270 \mathrm{~nm}$ ), the gel quickly bent in $1 \mathrm{~min}$. The gel ends moved toward the positive electrode as shown in Fig. 9b. During the bending, the center of gravity of the gel remained at the initial position. After the light was switched off, the gel slowly returned to the initial straight shape in the electric field.

In order to determine quantitatively the response time, one end of the rod-shaped gel was fixed to the wall and the distance that the free end moved from the initial position was measured as a function of irradiation time. Figure 10 shows the result. The free end moved toward the positive electrode with an initial speed of $0.40 \mathrm{~mm} / \mathrm{s}$. When the polarity of the electric field was changed, the end moved in the opposite direction. When the electric field was switched off, the bent gel returned to the initial position with a speed of $0.075 \mathrm{~mm} / \mathrm{s}$.

The bending rate depended on the applied field. Upon increasing the field, the response time increased in proportion to the root of the applied field. Although the bending rate became very slow, the bending motion was observed in a very weak field of $1.25 \mathrm{~V} / \mathrm{cm}$. In this case, effective voltage applied to the gel was only $0.25 \mathrm{~V}$.

As described above, the addition of salts to the external aqueous solution decreased the photostimulated dilation of the gels. On the contrary, the response time of the bending motion in the electric field was accelerated by the addition of salts. The acceleration rate depended on the size and valency of positive ions. By the addition of $2 \times 10^{-4} \mathrm{CaCl}_{2}$, the bending rate was accelerated as much as 12 times. The rod-shape gel, one end of which was fixed on the wall, vibrated in response to the alternating electric field $(0.5 \mathrm{~Hz})$ under ultraviolet irradiation. .

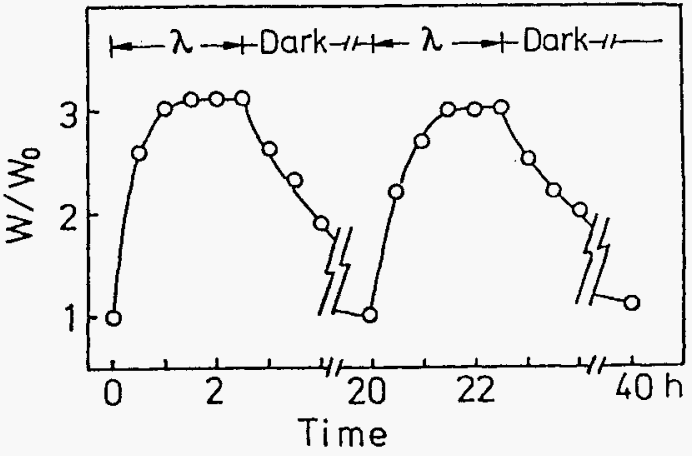

Fig. 8. Photostimulated volume change of polyacrylamide gel containing triphenylmethane leucohydroxide( 3.7 mols) with light of wavelength longer than $270 \mathrm{~nm}$. wo was the weight beforw photoirradiation. a)
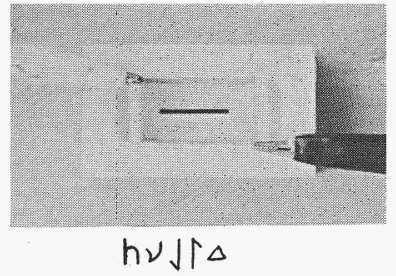

b)

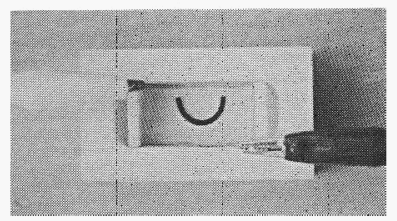

Fig. 9. Photostimulated bending motion of a rod-shaped acrylamide gel $(25 \mathrm{~mm}$ in length and $2 \mathrm{~mm}$ in diameter) containing $3.1 \mathrm{mols}$ triphenylmethane leucocyanide groups in an electric field $(10 \mathrm{~V} / \mathrm{cm})$. (a) before photoirradiation; (b) under UV irradiation. 
The bending behavior of the gel suggests inhomogeneous expansion of the gel in the electric field. The negative electrode side of the gel expands larger than the other side. Since the electric field is applied perpendicular to the gel axis, mobile negative ions are attracted to the positive electrode side in the gel. Consequently, excess positive charges are left on the other side. The large mobile ion concentration gradient on the gel surface is responsible to the gel bending. The thinness of the active layer is considered to play an important role to increase the response time.

\section{Photostimulated discontinuous volume change of polymer gels (ref. 20)}

By introducing the concept of photostimulated phase transition as shown in Fig. 2 to the gel system, it becomes possible to construct a sensitive photo-deformable gel. Poly (Nisopropylacrylamide) gels having triphenylmethane leucocyanide groups were synthesized in attempting to make a gel which shows a photostimulated volume phase transition. Figure 11 shows the equilibrium volume of the gels measured as a function of temperature, with and without ultraviolet irradiation, which induces the photo-dissociation of the leucocyanide groups. When the gel was not irradiated with ultraviolet light, the gel underwent a sharp, but continuous volume change at around $30.0^{\circ} \mathrm{C}$. Upon irradiation, the gel showed a discontinuous volume transition. The temperature was raised from $25^{\circ} \mathrm{C}$ gradually. At $32.6^{\circ} \mathrm{C}$, the volume of the gels suddenly decreased by approximately 10 times. Above the transition temperature, the gel volume did not change markedly. When the temperature was lowered starting from $35^{\circ} \mathrm{C}$, the gel swelled discontinuously by 10 times in volume at approximately $31.5^{\circ} \mathrm{C}$. The presence of the hysteresis confirms that this is the phase transition. When the temperature was fixed at $32.0^{\circ} \mathrm{C}$, the gel underwent a discontinuous swelling-shrinking switching upon irradiation and removing ultraviolet light.

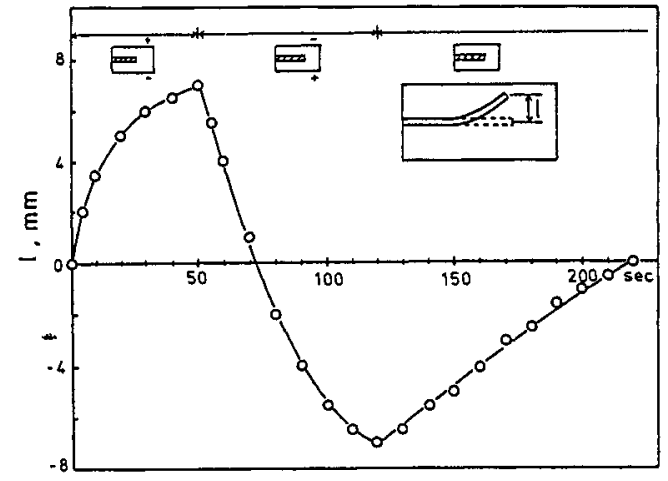

Fig. 10. Photostimulated bending of a rodshaped acrylamide gel containing 3.1 mols triphenylmethane leucocyanide groups in an electric field( $10 \mathrm{~V} / \mathrm{cm})$. The electric field was removed after $120 \mathrm{~s}$.

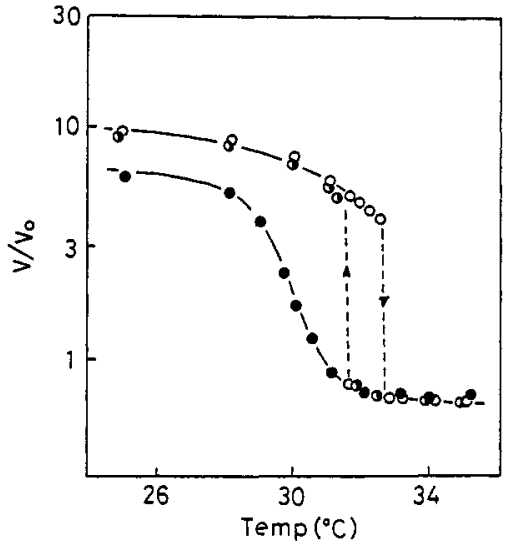

Fig. 11. The degree of swelling of poly(Nisopropylacrylamide) gel containing triphenylmethane leucocyanide groups $(0.8 \mathrm{~mol})$. $(\bullet)$ before photoirradiation; $(0)$ under UV irradiation in raising temperature; (0) under UV irradaition in lowering temperature.

\section{REFERENCES}

1. M. Irie, Adv. polym. Sci., 94, 27 (1990).

2. M. Irie, K. Hayashi, J. Macromol. Sci., Chem., A13, 511 (1979).

3. M. Irie, K. Hirano, S. Hashimoto, K. Hayashi, Macromolecules, 14, 262 (1981).

4. G.S. Kumar, D.C. Neckers, Chem. Rev., 89, 1915 (1989).

5. H.S. Blair, H.I. Pogue, J.E. Riordan, Polymer, 21, 1195 (1980).

6. G.S. Kumar, P. DePra, D.C. Neckers, Macromolecules, 17, 1912 (1984).

7. G.S. Kumar, P. DePra, K. Zhang, D.C. Neckers, Macromolecules, 17, 2463 (1984).

8. E.K. Zimmerman, J.K. Stille, Macromolecules, 14, 1246 (1985).

9. D. Kungwatchakun, M. Irie, Makromol. Chem. Rapid Comun. 9, 243 (1988).

10. M. Irie, H. Tanaka, Macromolecules, 16, 210 (1983).

11. M. Irie, w. Schnabel, Macromolecules, 18,394 (1985).

12. T.G. Fox, P.J. Flory, J.Am.Chem.SOC., 73, 1909, 1915 (1951).

13. M. Irie, T. Iwayanagi, Y. Taniguchi, Macromolecules, 18, 2418 (1985).

14. M. Irie, M. Hosoda, Makromol. Chem. Rapid Commun. $\overline{6}, 533$ (1985).

15. M. Irie, D. Kungwatchakun, Makromol. Chem. Rapid Commun., 5, 829 (1984).

16. M. Irie, D. Kungwatchakun, Macromolecules, 19, 2476 (1986).

17. A. Aviram, Macromolecules, 11, 1275 (1978).

18. M. Irie, Macromolecules, 19,2890 (1986).

19. M. Irie, Photophysics of Polymers, p107, ACS, Washington D.C. (1987).

20. A. Mamada, T. Tanaka, D. Kungwatchakun, M. Irie, Macromolecules, 23, 1517 (1990). 